\title{
CHARACTERIZATION OF TOTALLY UMBILICAL HYPERSURFACES
}

\author{
THOMAS HASANIS
}

\begin{abstract}
This paper gives a sufficient condition for a complete hypersurface of a Riemannian manifold of constant curvature to be umbilical. The condition will be given by an inequality which is established between the length of the second fundamental tensor and the mean curvature.
\end{abstract}

K. Nomizu and B. Smyth in [3] established a formula for the Laplacian of the second fundamental form of a hypersurface $M$ immersed with constant mean curvature in a space $\bar{M}$ of constant sectional curvature $c$. Later, M. Okumura in [4] characterized under certain conditions a totally umbilical hypersurface of a Riemannian manifold of nonnegative constant curvature by an inequality between the length of the second fundamental tensor and the mean curvature of the hypersurface.

In the present article we prove the following theorem.

TheOREM A. Let $M$ be an $n$-dimensional $(n>3)$ connected complete hypersurface immersed with constant mean curvature in an $(n+1)$-dimensional Riemannian manifold $\bar{M}$ of positive constant curvature $c$. If the second fundamental tensor $L$ satisfies

$$
\text { trace } L^{2}<\frac{1}{n-1}(\operatorname{trace} L)^{2}+2 c,
$$

then $M$ is a totally umbilical hypersurface and consequently a sphere.

REMARK. If $M$ is compact, Theorem A has been proved by Okumura in [4].

Finally we obtain the following result for complete minimal hypersurfaces of a sphere. In the compact case, this has been proved by J. Simons in [6].

THEOREM B. Let $M$ be a complete minimal hypersurface of the sphere $S^{n+1}(1 / \sqrt{c})$ of radius $1 / \sqrt{c}$. Then $\operatorname{Sup} S \geqslant c n$ or $M$ is totally geodesic.

1. Preliminaries. Let $\bar{M}$ be an $(n+1)$-dimensional Riemannian manifold of constant curvature $c$. Let $\varphi: M \rightarrow \bar{M}$ be an isometric immersion of an $n$-dimensional manifold $M$ into $\bar{M}$. In what follows we identify $M$ with $\varphi(M)$ and $p \in M$ with $\varphi(p) \in \varphi(M) \subset \bar{M}$. The tangent space $T_{p} M$ is also identified with a subspace of $T_{\varphi(p)} \bar{M}$. The Riemannian metric $g$ of $M$ is induced from the Riemannian metric

Received by the editors December 27, 1979 and, in revised form, February 26, 1980.

AMS (MOS) subject classifications (1970). Primary 53C40, 53C20.

Key words and phrases. Second fundamental tensor, totally umbilical hypersurfaces. 
$\bar{g}$ of $\bar{M}$ in such a way that

$$
g(X, Y)=\bar{g}(d \varphi(X), d \varphi(Y)),
$$

where $X, Y$ are vector fields of $M$.

We define the Weingarten map $L(X)=\nabla_{X} N$, where $N$ is a unit normal on $M$ and $\nabla$ the covariant differentiation in $\bar{M}$. The mean curvature $H$ of $M$ in $\bar{M}$ is defined by

$$
H=\frac{1}{n} \operatorname{trace} L .
$$

When at each point of $M$ we have trace $L^{2}=(1 / n)(\text { trace } L)^{2}, M$ is called a totally umbilical hypersurface of $\bar{M}$. In particular, if trace $L^{2}=0, M$ is called a totally geodesic hypersurface of $\bar{M}$. Now, we put

$$
Z X=L X-\frac{1}{n}(\operatorname{trace} L) X .
$$

Applying $Z$ to both members of (1.1), we get a globally defined linear transformation $Z^{2}$, where

$$
Z^{2} X=L^{2} X-\frac{2}{n}(\operatorname{trace} L) L X+\frac{1}{n^{2}}(\operatorname{trace} L)^{2} X .
$$

This implies that

$$
\operatorname{trace} Z^{2}=\operatorname{trace} L^{2}-\frac{1}{n}(\operatorname{trace} L)^{2} \text {, }
$$

because $Z$ is a symmetric linear transformation.

From equation (1.2) we conclude that trace $Z^{2}>0$ and equality holds everywhere iff $M$ is a totally umbilical hypersurface of $\bar{M}$.

Finally, we denote by $S$ the square of the norm of the second fundamental form of $M$ in $\bar{M}$ and by $R$ the scalar curvature of $M$. Then the following relations are well known

$$
\begin{aligned}
& S=\operatorname{trace} L^{2} \\
& R=n^{2} H^{2}-S+n(n-1) c .
\end{aligned}
$$

2. Main results. At first we state two lemmas, which are easy consequences of a result by Chen and Okumura [1], [2, p. 55].

LEMMA 2.1. Let $M$ be an $n$-dimensional hypersurface of a Riemannian manifold $\bar{M}$ of constant curvature $c$. If

$$
\text { trace } L^{2} \leqslant \frac{1}{n-1}(\operatorname{trace} L)^{2}+2 c \quad\left(\text { resp. trace } L^{2}<\frac{1}{n-1}(\operatorname{trace} L)^{2}+2 c\right)
$$

then the sectional curvatures of $M$ are nonnegative (resp. positive).

LEMMA 2.2. Let $M$ be a minimal hypersurface of the sphere $S^{n+1}(1 / \sqrt{c})$ of radius $1 / \sqrt{c}$. Assume that $S \leqslant c n-\varepsilon$, with $\varepsilon$ a constant number. Then the sectional curvatures of $M$ are bounded below by $(\varepsilon-(n-2)) c / 2$. 
Now, let $M$ be a complete hypersurface with constant mean curvature $H$ in a Riemannian manifold $\bar{M}$ with constant curvature $c$. We use the linear transformation $Z$ of $\S 1$ and set $f^{2}=\operatorname{trace} Z^{2}$. Obviously $f^{2}>0$ and $f^{2}=0$ everywhere on $M$ iff $M$ is totally umbilical. We repeat the same calculations as $M$. Okumura in [4] and get

$$
\begin{aligned}
\frac{1}{2} \Delta f^{2} \geqslant & f^{2}\left\{c n+\frac{1}{n}(\operatorname{trace} L)^{2}-\frac{n-2}{\sqrt{n(n-1)}}|\operatorname{trace} L| f-f^{2}\right\} \\
& +g(\nabla L, \nabla L) .
\end{aligned}
$$

Now, we proceed to the proofs of Theorems $A$ and $B$.

Proof of Theorem A. From the assumption of the theorem and relation (1.2) we get

$$
f^{2}<\frac{1}{n(n-1)}(\operatorname{trace} L)^{2}+2 c .
$$

We set

$$
\varepsilon_{1}=(n-2)\left\{c+\frac{(\operatorname{trace} L)^{2}}{n(n-1)}-\left[\left(c+\frac{(\operatorname{trace} L)^{2}}{n(n-1)}\right)^{2}-c^{2}\right]^{1 / 2}\right\} .
$$

Now, since $n>2$ and $c>0$ there exists a positive number $\varepsilon_{0}$, such that

$$
0<\varepsilon_{0} \leqslant \varepsilon_{1}=(n-2)\left\{c+\frac{(\operatorname{trace} L)^{2}}{n(n-1)}-\left[\left(c+\frac{(\operatorname{trace} L)^{2}}{n(n-1)}\right)^{2}-c^{2}\right]^{1 / 2}\right\} \text {. }
$$

Then

$$
\varepsilon_{0}^{2}-2(n-2)\left\{c+\frac{1}{n(n-1)}(\operatorname{trace} L)^{2}\right\} \varepsilon_{0}+c^{2}(n-2)^{2}>0
$$

or

$$
\frac{(\operatorname{trace} L)^{2}}{2(n-1)}+c-\frac{\varepsilon_{0}}{n-2}>\frac{|\operatorname{trace} L|}{\sqrt{n(n-1)}}\left[\frac{n}{4(n-1)}(\operatorname{trace} L)^{2}+c n-\varepsilon_{0}\right]^{1 / 2}
$$

or

$$
\begin{aligned}
{\left[\frac{(\operatorname{trace} L)^{2}}{n(n-1)}+2 c\right]^{1 / 2}<} & -\frac{(n-2)}{2 \sqrt{n(n-1)}}|\operatorname{trace} L| \\
& +\left\{\frac{n}{4(n-1)}(\operatorname{trace} L)^{2}+c n-\varepsilon_{0}\right\}^{1 / 2} .
\end{aligned}
$$

From (2.2) and the last inequality we obtain

$$
0 \leqslant f \leqslant-\frac{n-2}{2 \sqrt{n(n-1)}}|\operatorname{trace} L|+\left\{\frac{n}{4(n-1)}(\operatorname{trace} L)^{2}+c n-\varepsilon_{0}\right\}^{1 / 2}
$$

or

$$
f^{2}+\frac{(n-2)}{\sqrt{n(n-1)}}|\operatorname{trace} L| f-c n-\frac{1}{n}(\operatorname{trace} L)^{2}+\varepsilon_{0}<0
$$


and thus

$$
c n+\frac{1}{n}(\operatorname{trace} L)^{2}-\frac{(n-2)}{\sqrt{n(n-1)}}|\operatorname{trace} L| f-f^{2}>\varepsilon_{0} .
$$

So, by using (2.3) the inequality (2.1) gives

$$
\frac{1}{2} \Delta f^{2} \geqslant f^{2} \varepsilon_{0}+g(\nabla L, \nabla L) \geqslant f^{2} \varepsilon_{0} .
$$

Since $f^{2}$ is bounded above and the sectional curvatures of $M$ are bounded below (see Lemma 2.1), we claim that $f^{2}=0$ everywhere on $M$. In fact, if, for a point $p_{1}$ we had $f^{2}\left(p_{1}\right)=b>0$, then from (2.4) we should have $\frac{1}{2} \Delta f^{2}\left(p_{1}\right)>b \varepsilon_{0}=$ const $>$ 0 , and thus for all points $q \in M$ such that $f^{2}(q)>f^{2}\left(p_{1}\right)$ ought to have $\frac{1}{2} \Delta f^{2}(q)>$ $b \varepsilon_{0}=$ const $>0$, which contradicts Omori's theorem $A^{\prime}$ in [5]. So $f^{2}=0$ everywhere on $M$ and the proof is finished.

Now, let $M$ be a complete minimal hypersurface of the sphere $S^{n+1}(1 / \sqrt{c})$ of radius $1 / \sqrt{c}$. Then $(2.1)$ becomes

$$
\frac{1}{2} \Delta S \geqslant S(c n-S)+g(\nabla L, \nabla L) .
$$

Proof of Theorem B. It is sufficient to prove the following: If Sup $S<c n$, then $M$ must be totally geodesic. In fact, if $\operatorname{Sup} S<c n$, then there exists a positive number $\varepsilon$ such that $S \leqslant c n-\varepsilon$ everywhere on $M$. Then using Lemma 2.2 we conclude that $M$ has sectional curvatures bounded below. Also $c n-S>\varepsilon$ and (2.5) gives

$$
\frac{1}{2} \Delta S \geqslant S \varepsilon \text {. }
$$

Now, we proceed as in the proof of Theorem $\mathrm{A}$ and we conclude that $S=0$ everywhere on $M$, i.e. $M$ is totally geodesic.

\section{REFERENCES}

1. B-Y. Chen and M. Okumura, Scalar curvature, inequality and submanifold, Proc. Amer. Math. Soc. 38 (1973), 605-608.

2. B-Y. Chen, Geometry of submanifolds, Marcell Dekker, New York, 1973.

3. K. Nomizu and B. Smyth, A formula of Simon's type and hypersurfaces with constant mean curvature, J. Differential Geom. 3 (1969), 367-377.

4. M. Okumura, Hypersurfaces and a pinching problem on the second fundamental tensor, Amer. J. Math. 96 (1974), 207-213.

5. H. Omori, Isometric immersions of Riemannian manifolds, J. Math. Soc. Japan 19 (1967), $205-214$.

6. J. Simons, Minimal varieties in Riemannian manifolds, Ann. of Math. (2) 88 (1968), 62-105.

Department of Mathematics, University of Ionnnina, Ionnnina, Greece 